\title{
Cirugía urgente y electiva en ortopedia y traumatología durante la pandemia de COVID-19
}

\author{
Urgent and elective surgery in orthopedics and traumatology \\ during the COVID-19 pandemic
}

\author{
Jesús Rodríguez López* \\ * Médico Especialista en Ortopedia y Traumatología del Centro de Investigación y Docencia en \\ Ciencias de la Salud de la Universidad Autónoma de Sinaloa en Culiacán, Sinaloa. \\ Correspondencia: Dr. Jesús Rodríguez López. Mar de Plata No. 2600-51, Col. Maralago, C.P. 80014, \\ Culiacán, Sinaloa. Correo electrónico: dr_jesus_rodriguez_lopez@yahoo.com.mx
}

\section{RESUMEN}

Durante la emergencia de la pandemia por COVID-19, el impacto en la cirugía ortopédica en México no ha sido distinto a otros sitios del mundo; en nuestro país contamos con recursos limitados ante esta enfermedad y el efecto que tendrá en nuestro sistema de salud será quizá más severo. En el presente artículo se destacan cuáles son los procedimientos quirúrgicos en ortopedia y traumatología que no podrán diferirse porque se pone en peligro la vida y/o la función en los pacientes, además de la importancia que en los servicios de cirugía ortopédica se priorice la atención de las lesiones.

Palabras clave: COVID-19, enfermedad por coronavirus, ortopedia, traumatología, cirugía.

\section{ABSTRACT}

The impact COVID-19 emergency pandemic has had within the orthopedic surgery, is no different in Mexico, than in the rest of the world. The only distinction is that Mexico has limited resources against the coronavirus disease, which will perhaps cause a more severe impact on our health system. The aim of this article is to highlight the surgical procedures in orthopedics and traumatology that cannot be deferred because life and/or functions of the patients might be endangered. In addition, it will also discuss the importance of the role that orthopedic surgery services have in prioritizing the injurie treatment.

Keywords: COVID-19, coronavirus disease, orthopedics, trauma, surgery.

\section{INTRODUCCIÓN}

Como enfermedad emergente la Organización Mundial de la Salud (OMS) declaró como pandemia a la enfermedad por COVID-19 el 11 de marzo de $2020^{1}$ y en las siguientes dos semanas la enfermedad por coronavirus se diseminó a 330,000 personas y causó 13,700 fallecimientos. ${ }^{2}$ 
El objetivo del presente artículo es revisar como se ha enfrentado en los diferentes continentes el manejo quirúrgico de la patología musculoesquelética y las prioridades para su atención en este tiempo sin precedentes como es esta pandemia.

\section{URGENCIAS QUIRÚRGICAS}

Se considera cirugía electiva todo aquel procedimiento que se requiere para tratar problemas crónicos y no causa un daño significativo al paciente si se difiere, estas cirugías deben programarse hasta pasar la contingencia sanitaria. Algunos ejemplos de estas cirugías son: reemplazo articular, ${ }^{3}$ artrodesis de la columna vertebral, afecciones crónicas atraumáticas de las articulaciones, así como lesiones meniscales degenerativas, rupturas crónicas del manguito rotador, síndrome del túnel del carpo, cirugías por tumores musculoesqueléticos benignos no agresivos o benignos agresivos sin fractura. ${ }^{4}$ En los pacientes pediátricos se consideran electivos los retiros de implantes, osteotomías por displasia de cadera en adolescentes, corrección de deformidades angulares, caderas luxadas en mayores de un año, tenotomías y/o alargamientos músculo-tendinosos en pacientes con parálisis cerebral, corrección de deformidades de extremidades superiores y/o inferiores, tratamiento quirúrgico de la escoliosis, etcétera. A medida que avanzan los contagios por COVID-19 se deben organizar los servicios para considerar el manejo conservador de las lesiones y considerar la cirugía ambulatoria en algunos pacientes con displasia de cadera menores de un año, deslizamiento de la cabeza femoral, reducción de cadera paralítica, biopsia de tumores claramente benignos, corrección de mala unión y tratamiento quirúrgico de osteocondritis disecante.

Se considera cirugía urgente todo aquel procedimiento que no puede ser diferido porque se pone en riesgo la vida del paciente o la función de un órgano o extremidad como son: las fracturas expuestas, síndrome compartamental, fracturas inestables de pelvis, fracturas articulares acompañadas de compromiso neurológico o vascular, fracturas patológicas, politrauma, biopsias de tumores probablemente malignos, cirugías definitivas en pacientes con tumores malig-

Tabla 1: Procedimientos quirúrgicos ortopédicos urgentes.

Artritis piógena

Fracturas expuestas (II y III clasificación de Gustilo)

Síndrome de cauda equina

Traumatismo raquimedular con afectación neurológica

Síndrome compartamental

Fractura instable de pelvis con datos de compromiso hemodinámico

Fracturas articulares acompañadas de compromiso vascular y/o neurológico

Luxaciones (de cualquier articulación o de una prótesis)

Mano o pie severamente fracturado

Lesiones traumáticas expuestas agudas (que involucren lesiones tendinosas)

Lesiones traumáticas sin fracturas acompañadas de afectación vascular y/o neurológica

Biopsias y tratamientos de tumores malignos o con fracturas patológicas 
nos al término de ciclos de quimioterapia, luxaciones, mano o pie severamente lesionado, lesiones traumáticas expuestas agudas, lesiones traumáticas agudas sin fracturas acompañadas de afectación vascular y/o nerviosa, pie diabético complicado, síndrome de cauda equina, trauma raquimedular con afectación neurológica e infecciones agudas (Tabla 1). ${ }^{4-6}$ En el paciente pediátrico se deberán tratar como urgentes las osteosíntesis de fracturas de fémur, tibia, reducción y fijación de fracturas supracondíleas humerales, cóndilo lateral humeral, fracturas de antebrazo en adolescentes, biopsia de tumor aparentemente maligno, amputaciones de extremidades necróticas, politrauma, sepsis por osteomielitis, artritis séptica, absceso subperióstico, fracturas expuestas Gustilo II y III, síndrome compartamental, luxaciones agudas y fracturas con compromiso vascular.

Ante el constante cambio de las Guías de tratamiento de la enfermedad por coronavirus, al ser emergente y ante la necesidad de priorizar los recursos con los que se cuentan (que son escasos), se requiere de la organización de los Servicios de Cirugía Ortopédica para enfrentar de la mejor manera esta contingencia sanitaria y la experiencia de los sistemas de salud en el mundo se van restringiendo en la asignación de los recursos, limitando la cirugía electiva y realizando los procedimientos quirúrgicos urgentes. ${ }^{7,8}$

\section{BIBLIOGRAFÍA}

1. Cucinotta D, Vanelli M. WHO declares COVID-19 a pandemic. Acta Biomed. 2020; 91: 157-160.

2. Coronavirus: A visual guide to the pandemic. [On line march 22, 2020] Available in: https://www.bbc. com/news/world-51235105.

3. Scott CE, MacDonald DJ, Howie CR. "Worst than death" and waiting for a joint arthroplasty. Bone Joint J. 2019; 101-B: 941-950.

4. American College of Surgeons. COVID-19 Guidelines for triage of orthopaedic patients. [On line march 20, 2020] Available in: https://www.facs.org/covid-19/clinical-guidance/elective-case/orthopaedics.

5. British Orthopaedic Association. Management of patients with urgent orthopaedic conditions and trauma during the coronavirus pandemic. [On line march 24, 2020] Available in: https://www.boa.ac.uk/resources/ covid-boasts-combined.html.

6. Asociación Española de Cirujanos: Recomendaciones generales de la atención de la patología quirúrgica urgente en el contexto de la pandemia por COVID-19 https://www.aecirujanos.es/files/noticias/152/ documentos/Recomendaciones_AEC_en_CIRUGIA_DE_URGENCIASv2.pdf

7. American College of Surgeons Committee on Trauma: Maintaining trauma center access \& care during the COVID-19 pandemic: Guidance document for trauma medical directors. [On line march 20, 2020] Available in: https://www.facs.org/quality-programs/trauma/maintaining-access.

8. Liang ZC, Wang W, Murphy D, Hoi Po Hui J. Novel coronavirus and orthopaedic surgery: early experiences form Singapore. J Bone Joint Surg Am. 2020; e000236. doi: 102106/JBJS.20.00326. 